\title{
Sulfur/DABCO Promoted Reductive Coupling/Annulation Cascade Re- action between o-Hydroxy/Amino Nitrobenzenes and Benzaldehydes
}

\author{
Minh-Huy Dinh Danga,b \\ Linh Ho Thuy Nguyen ${ }^{b}$ \\ Phuong Hoang Tran*a \\ a Department of Organic Chemistry, Faculty of Chemistry, University \\ of Science, Vietnam National University, Ho Chi Minh City (VNU- \\ HCM) 721337, Vietnam \\ thphuong@hcmus.edu.vn \\ ${ }^{b}$ Center for innovative Materials and Architectures, Vietnam National \\ University, Ho Chi Minh City 721337, Vietnam
}

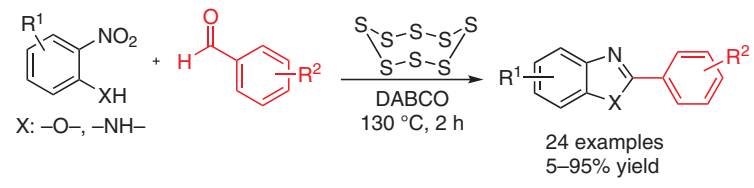

using superacid hafnium-based metal-organic frameworks ${ }^{8}$ or phosphonium acidic ionic liquids ${ }^{9}$ as catalysts. This approach suffers from a lack of commercially available starting materials and requires expensive catalyst, which prevents it from being applied in large-scale processes. Recently, a AgPd alloy and defected perovskite-type $W_{2.72}$ nanorods were used for efficient one-pot conversion of nitrophenol/aldehyde into benzoxazole using formic acid as hydrogen source (Scheme 1). ${ }^{10}$ However, the protocol suffers from drawbacks such as expensive and unavailable catalyst, and the addition of additives and volatile organic solvent. We report herein a novel, simple, and efficient method for the synthesis of benzoxazoles and benzimidazoles from 2-nitrophenols or 2-nitroanilines and benzaldehydes using available and inexpensive sulfur/DABCO as the catalyst. The prominent features of the current method are simplicity, inexpensive and readily available starting materials, and a broad scope of substrates.

Initially, we envisioned that treatment of sulfur with DABCO could give the zwitterion $\mathbf{A}$, which would allow condensation of $o$-nitrophenol with aldehydes and the formation of benzoxazole scaffolds. To check this hypothesis, we used the sulfur/DABCO catalyst for the redox condensation between $o$-nitrophenol with benzaldehyde, without a hydrogen source, at $130{ }^{\circ} \mathrm{C}$. $o$-Nitrophenol and benzaldehyde were chosen as model substrates to establish the optimum reaction conditions. In the absence of sulfur and/or DABCO, the desired benzoxazole was not formed. Interestingly, when sulfur was activated with DABCO, benzoxazole was obtained in good to excellent yields (Table 1).

The reaction was best performed at $130{ }^{\circ} \mathrm{C}$ in sulfur/ DABCO as an activator (Table 1, entry 7). Conducting the reaction at higher temperature resulted in decomposition of the product (entry 13). It should be noted that to obtain benzoxazoles from $o$-nitrophenols and aldehydes, the expensive catalyst (AgPd nanoparticles in the presence of 
(a) Acid-catalyzed condensation of $o$-aminophenol with aromatic aldehydes ${ }^{7}$

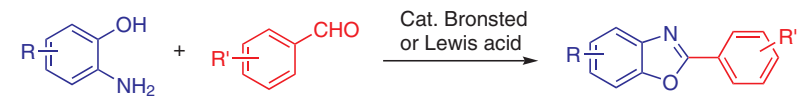

(b) $\mathrm{Ag}_{48} \mathrm{Pd}_{52} / \mathrm{WO}_{2.72}$-catalyzed redox condensation of $o$-nitrophenol with aromatic aldehydes ${ }^{10}$

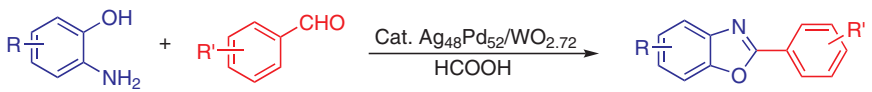

(c) Sulfur/DABCO-promoted redox condesation of o-nitrophenol with aromatic aldehydes (this work)

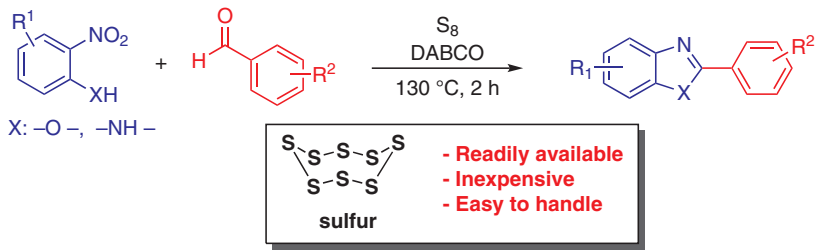

Scheme 1 Synthetic routes to acid catalysts (a), $\mathrm{Ag}_{48} \mathrm{Pd}_{52} / \mathrm{WO}_{2.72}$ (b), and our work (c) on the synthesis of 2-arylbenzoxazoles

$\mathrm{WO}_{2.72}$ nanorods) and formic acid as a hydrogen source for hydrogenation reactions were previously required. ${ }^{10}$ Most significantly, in this report, inexpensive and readily available sulfur/DABCO reagents were sufficient to achieve full conversion of 0 -nitrophenols into benzoxazoles.

Table 1 Optimization of the Reaction Conditions ${ }^{\mathrm{a}}$

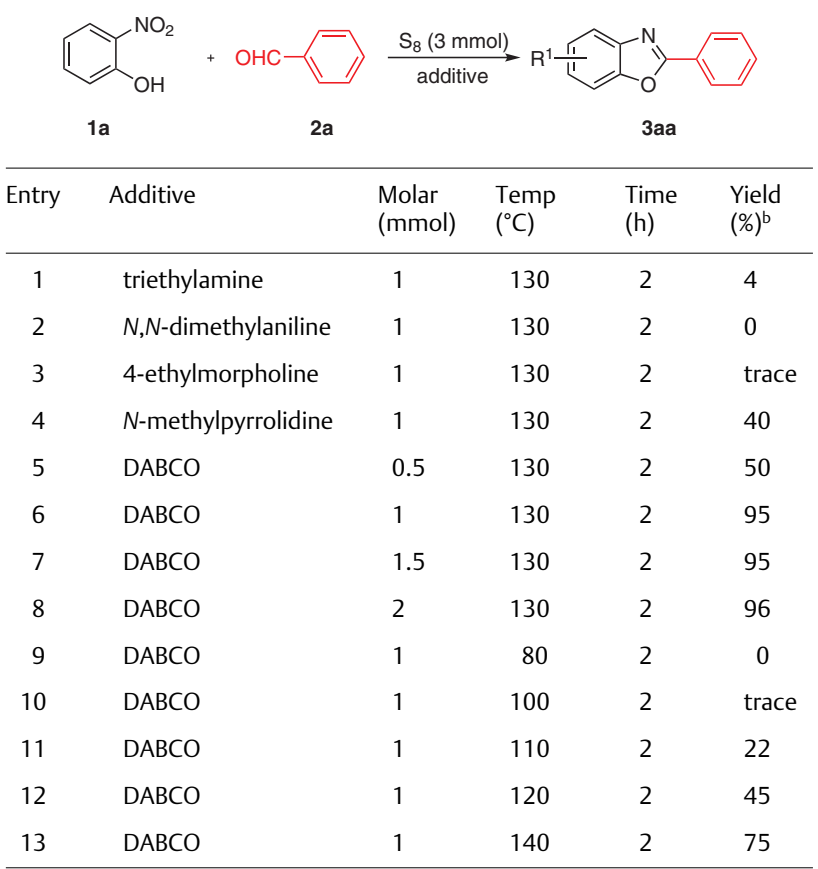

a Reaction conditions: $1 \mathbf{a}(1 \mathrm{mmol}), \mathbf{2 a}(1 \mathrm{mmol}), \mathrm{S}$ (3 mmol, $96 \mathrm{mg})$. ${ }^{\mathrm{b}} \mathrm{GC}$ yield.
Once the optimal conditions had been identified, the scope of the reaction was examined with 24 examples, as summarized in Scheme 2. The reactions of aromatic aldehydes with 0 -nitrophenol proceeded smoothly with good to excellent yields (3aa-aj). Various functional benzaldehydes such as methyl, tert-butyl, fluoro, chloro, or methoxy at the $p$-position were found to be suitable substrates for the optimized transformation (3aa-af). However, when 0 fluorobenzaldehyde was used, the amount of desired product 3ag was greatly reduced due to the formation of $3 \mathrm{H}$ benzo[c][1,2]dithiole-3-thione (detected by NMR and MS analyses) as a by-product (see the proposed mechanism below). Similar behavior was found for 2-chlorobenzaldehyde, which afforded the desired product in low yield (3ah). However, 2-hydroxybenzaldehyde was reactive under the current conditions and the desired product was afforded in up to $95 \%$ GC yield. Heterocyclic aldehydes such as pyridine-4-carbaldehyde were also reactive (3aj, $\mathbf{3 b i}$, and $\mathbf{3 c i}$ ). When 5-methyl-2-nitrophenol was used, the desired products were obtained in lower yields even when the reaction time was extended to five hours (3ba, $\mathbf{3 b b}, \mathbf{3 b e}, \mathbf{3 b \mathbf { b }}$, and 3bi). A possible explanation for the decrease of yields was the inductive effect of the methyl group, which decreases the reactivity of the nitro group. Interestingly, 5-chloro-2nitrophenol was successfully condensed under the current conditions, and the expected products 3ca-ci were obtained in good yields. The benzimidazole products could be formed exclusively in moderate to good yield in the reaction. The reaction of $\boldsymbol{o}$-nitrophenol with benzaldehyde was also carried out on a $10 \mathrm{mmol}$ scale, and the yield was almost the same as that on the $1 \mathrm{mmol}$ scale ( $86 \%$ vs. $90 \%$ ). 

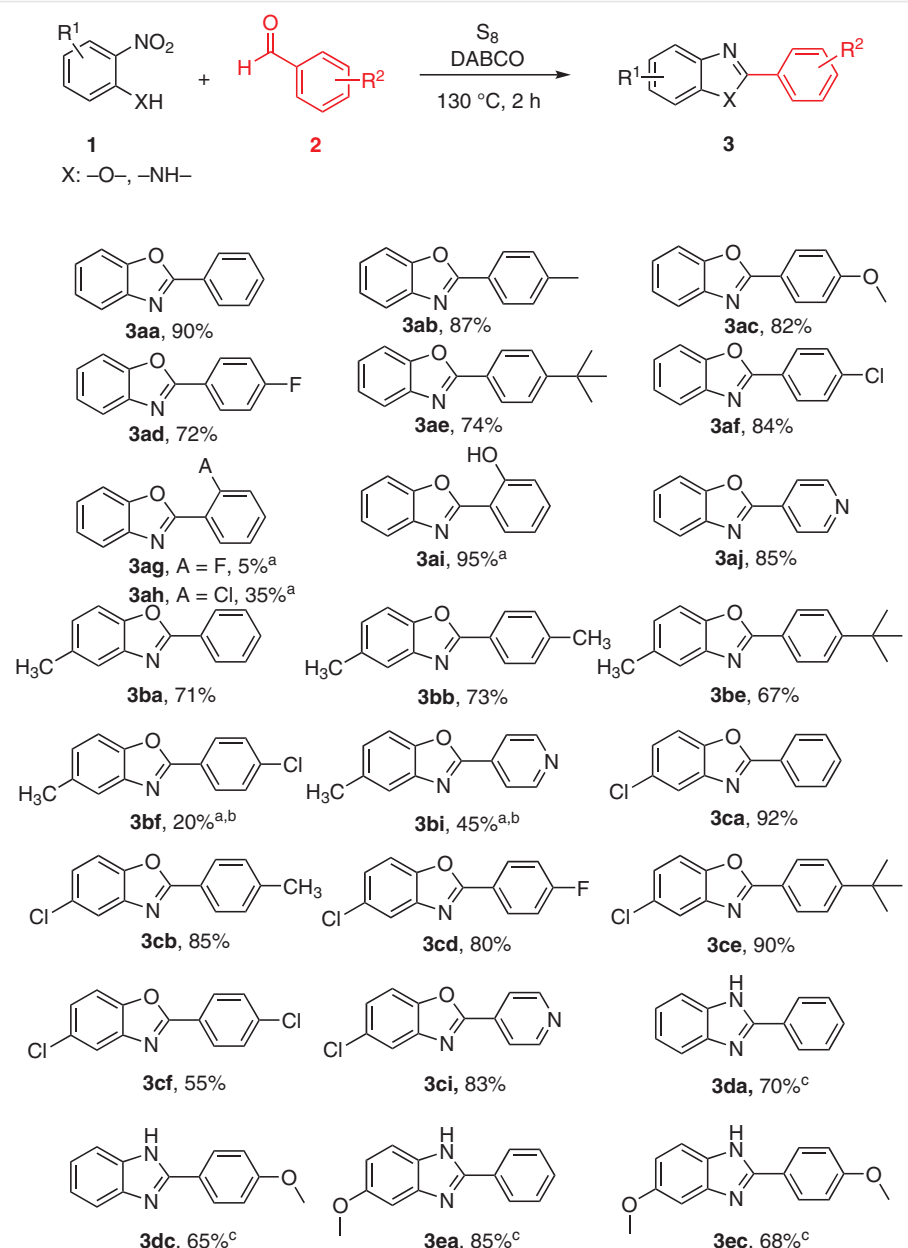

Scheme 2 Reaction scope and versatility. Reagents and conditions: 1 ( $1 \mathrm{mmol}), 2$ (1 mmol), S ( $3 \mathrm{mmol}, 96 \mathrm{mg})$, DABCO $(1 \mathrm{mmol}), 130{ }^{\circ} \mathrm{C}, 2 \mathrm{~h}$. Yields refer to isolated products unless otherwise noted. ${ }^{\text {a }}$ Yields were determined by GC. ${ }^{\mathrm{b}} 5 \mathrm{~h}$. ${ }^{\mathrm{c}} 10 \mathrm{~h}$.

To further understand the reaction pathway for the synthesis of benzoxazole, control experiments were carried out under the optimized conditions. First, each couple of starting materials was heated together. Reaction of sulfur with 0 -nitrophenol and of $o$-nitrophenol with benzaldehyde did not proceed (based on TLC or GCMS analysis). In contrast, when sulfur was treated with DABCO, a higher yield of benzoxazole was observed. Although the mechanism of the reaction is not clear at this stage, a proposed mechanism is presented in Scheme 3. In this scheme the reaction is suggested to be initiated by attack of sulfur on $\mathrm{DABCO}$ to form zwitterion A. Subsequent attack of this precursor on benzaldehyde leads to $\mathbf{C}$, which reacts with $\boldsymbol{o}$-nitrophenol to afford nitro polysulfide $\mathbf{D}^{\text {.c }}$ Subsequent fragmentation of $\mathbf{D}$ leads to $\boldsymbol{o}$-nitrophenyl benzoate $\mathbf{E}$, which undergoes deoxygenation to give 2-nitrosophenyl benzoate F. Although the detailed mechanism of the transformation of $\mathbf{F}$ into benzoxazole is not clear at the current time, the sulfonation and annulation process can occur from the nitroso group and carbonyl in benzoate to form intermediate
H. ${ }^{11}$ The elimination of $\mathrm{SO}_{3}$ molecule of $\mathrm{H}$ will give the benzoxazole. Furthermore, based on our experiments and on previous reports, a mechanism of byproduct formation with halobenzaldehydes is proposed in Scheme 4.

In general, we believe that our protocol is more convenient, simpler and cheaper than previous protocols for the synthesis of benzoxazoles, which certainly required more expensive/complex catalysts, an inert atmosphere, and reducing agents or solvents. Moreover, unlike most metalcatalyzed reactions involving molecular hydrogen or other stronger reductants, which are generally not compatible with the aldehyde functional group, our selective reduction method can be carried out in the presence of different $o$-nitrophenols and $o$-nitroanilines to provide 2-arylbenzoxazoles and benzimidazoles under green conditions.

In conclusion, we have developed a new, remarkably simple, and efficient method for the direct redox condensation of $o$-nitrophenols/anilines with aromatic aldehydes using elemental sulfur in DABCO under solvent- and catalyst- 


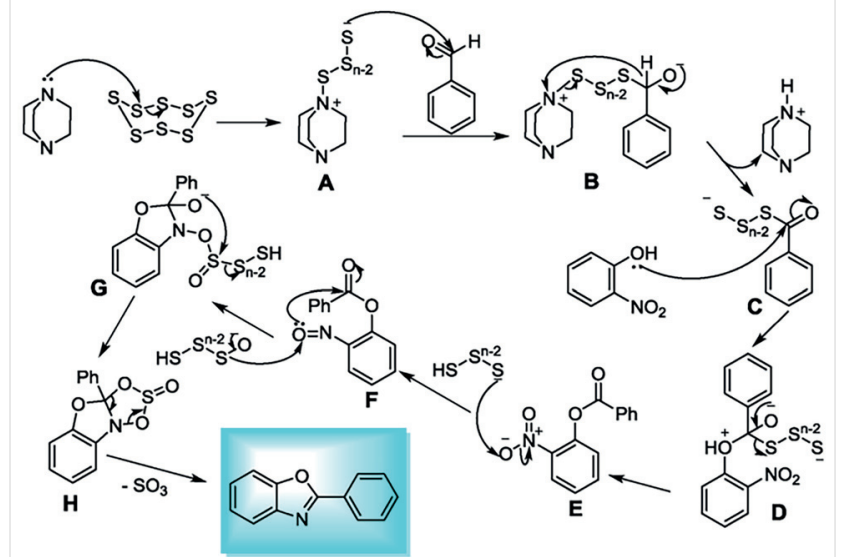

Scheme 3 Proposed mechanism

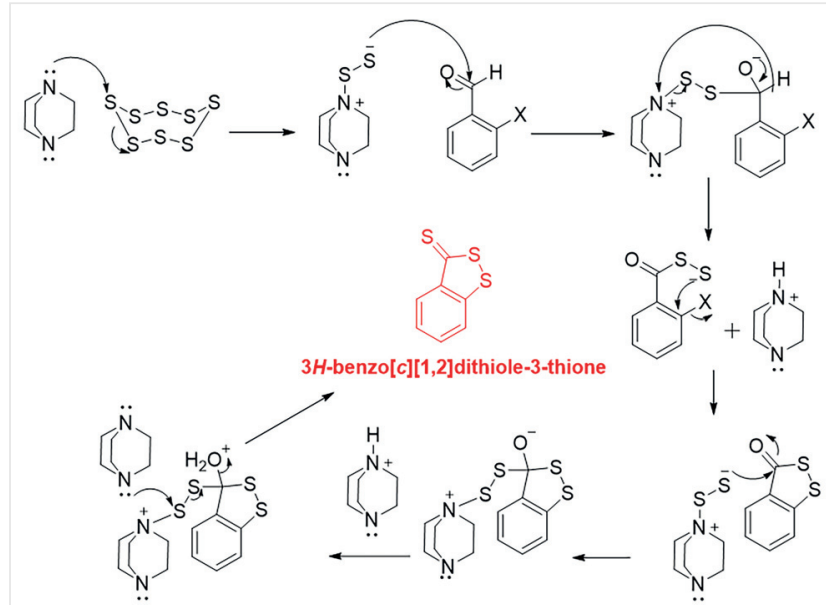

Scheme 4 Proposed mechanism for by-product formation with substitution of halo groups at the ortho-position

free conditions. This strategy is capable of providing a wide range of 2-arylbenzoxazoles and 2-arylbenzimidazoles, is highly versatile, and has great potential for industrial application. Moreover, the present method is highlighted for the simplicity and efficiency in affording benzoxazoles and benzimidazoles from simple, available and inexpensive starting materials. Further studies on the mechanism and scope of the reaction are in progress.

\section{Materials}

Sulfur (purity $\geq 99.5 \%$ ), triethylamine (purity $\geq 98 \%$ ), $N$-methylpyrolidine (purity $\geq 98 \%$ ), 1,4-diazabicyclo[2.2.2] octane (purity $\geq 97 \%$ ), 4ethylmorpholine (purity $\geq 97 \%$ ), $N, N$-dimethylaniline (purity $\geq 98 \%$ ), 2-nitrophenol (purity $\geq 97 \%$ ), 4-methyl-2-nitrophenol (purity $\geq 99 \%$ ), 4-chloro-2-nitrophenol (purity $\geq 97 \%$ ), 2-nitroaniline (purity $\geq 98 \%$ ), 5-methoxy-2-nitroaniline (purity $\geq 97 \%$ ), benzaldehyde (purity $\geq$ 99\%), 4-methylbenzaldehyde (purity $\geq 97 \%$ ), 4-methoxybenzaldehyde (purity $\geq 98 \%$ ), 4-fluorobenzaldehyde (purity $\geq 97 \%$ ), 4-chlorobenzaldehyde (purity $\geq 98 \%$ ), 4-tert-butylbenzaldehyde (purity $\geq 95 \%$ ), 2-fluorobenzaldehyde (purity $\geq 97 \%$ ), 2-chlorobenzaldehyde (purity $\geq$
97\%), 2-hydroxybenzaldehyde (purity $\geq 99 \%$ ), 4-pyridinecarboxaldehyde (purity $\geq 97 \%$ ) were purchased from Sigma-Aldrich. Silica gel 230-400 mesh for flash chromatography, TLC plates (silica gel 60 F254), ethyl acetate (anhydrous, purity $\geq 99 \%$ ), acetone and $n$-hexane (anhydrous, purity $\geq 99.5 \%$ ) were obtained from Merck. Deuterated solvents, $\mathrm{CDCl}_{3}$, DMSO- $d_{6}$ and acetone- $d_{6}$, were purchased from Cambridge Isotope Laboratories (Andover, MA). All chemicals were used without further purification.

\section{Equipment and analytical methods}

The chemicals were measured by using an Ohaus 80251621 explorer precision balance. The reactions were conducted on an IKA C-MAG HS7 magnetic stirrer. The products were analyzed by thin-layer chromatography (TCL) performed on F-254 silica gel coated aluminum plates from Merck. Column chromatography was performed on silica gel 60, 0.04-0.06 mm (230-400 mesh). Melting points were recorded with a Buchi B-545 melting point Apparatus.

Fourier transform infrared (FT-IR) spectra were measured on a Bruker E400 FT-IR spectrometer using potassium bromide pellets. Nuclear magnetic resonance $\left({ }^{1} \mathrm{H}\right.$ and ${ }^{13} \mathrm{C}$ NMR $)$ spectra were acquired on a Bruker advance II $500 \mathrm{MHz}$ NMR spectrometer. Chemical shifts were quoted in parts per million ( $\mathrm{ppm}$ ) and referenced to the appropriate solvent peak. Gas chromatography-mass spectrometry (GC-MS) measurements were carried out on an Agilent GC System 7890 equipped with a mass selective detector (Agilent 5973N) and a capillary DB5MS column $(30 \mathrm{~m} \times 250 \mu \mathrm{m} \times 0.25 \mu \mathrm{m})$.

\section{General Procedure}

To a round-bottomed flask equipped with a magnetic stirrer was sequentially added $o$-nitrophenol or $o$-nitroaniline $(1 \mathrm{mmol})$, aldehyde ( $1 \mathrm{mmol})$, sulfur (3 mmol, $96 \mathrm{mg})$, and DABCO (1,4-diazabicyclo[2.2.2] octane; $1 \mathrm{mmol}, 112 \mathrm{mg}$ ). The reaction mixture was stirred at $130{ }^{\circ} \mathrm{C}$ for $2 \mathrm{~h}$ under air. Upon completion, the reaction mixture was dissolved in EtOAc, and the residual sulfur was removed. The crude mixture was purified by a silica gel column (petroleum ether/EtOAc, from 95:5 to $90: 10$ ) to give the expected product.

\section{2-Phenylbenzoxazole (3aa)}

Yield: 90\% (175 mg); analytical TLC on silica gel (9:1 $n$-hexane/ EtOAc); white solid; $\mathrm{mp} 102-103.5^{\circ} \mathrm{C}$.

FT-IR (KBr): 3059, 2925, 2854, 1775, 1615, 1551, 1475, 1448, 1285 , $1240 \mathrm{~cm}^{-1}$.

${ }^{1} \mathrm{H}$ NMR $\left(500 \mathrm{MHz}, \mathrm{CDCl}_{3}\right): \delta=8.28-8.26(\mathrm{~m}, 2 \mathrm{H}), 7.80-7.77(\mathrm{~m}, 1 \mathrm{H})$, 7.60-7.58 (m, $1 \mathrm{H}), 7.55-7.52$ ( $\mathrm{m}, 3 \mathrm{H}), 7.38-7.34$ (m, $2 \mathrm{H}$ ).

${ }^{13} \mathrm{C}$ NMR $\left(125 \mathrm{MHz}, \mathrm{CDCl}_{3}\right): \delta=163.2,150.9,142.2,131.7,129.0$, $127.8,127.3,125.3,124.7,120.1,110.7$.

GC-MS (EI, $70 \mathrm{eV}): m / z=195[\mathrm{M}]^{+}$.

\section{2-(4-Methylphenyl)benzoxazole (3ab)}

Yield: $87 \%$ (181 mg); analytical TLC on silica gel (9:1 $n$-hexane/ EtOAc); white solid; mp $113-114.5^{\circ} \mathrm{C}$.

FT-IR (KBr): 3056, 2920, 2854, 1728, 1620, 1554, 1499, 1450, $1242 \mathrm{~cm}^{-1}$.

${ }^{1} \mathrm{H}$ NMR $\left(500 \mathrm{MHz}, \mathrm{CDCl}_{3}\right): \delta=8.15(\mathrm{~d}, J=8.0 \mathrm{~Hz}, 2 \mathrm{H}), 7.77-7.75(\mathrm{~m}$, $1 \mathrm{H}), 7.58-7.56(\mathrm{~m}, 1 \mathrm{H}), 7.35-7.32(\mathrm{~m}, 4 \mathrm{H}), 2.44(\mathrm{~s}, 3 \mathrm{H})$.

${ }^{13} \mathrm{C}$ NMR $\left(125 \mathrm{MHz}, \mathrm{CDCl}_{3}\right): \delta=163.5,150.8,142.3,142.2,129.8$, $127.8,125.1,124.7,124.5,120.0,110.6,21.8$.

GC-MS (EI, $70 \mathrm{eV}): m / z=209[\mathrm{M}]^{+}$. 


\section{2-(4-Methoxyphenyl)benzoxazole (3ac)}

Yield: $82 \%$ (184 mg); analytical TLC on silica gel (9:1 $n$-hexane/ EtOAc); white solid; $\mathrm{mp} 120-122{ }^{\circ} \mathrm{C}$

FT-IR (KBr): 3050, 2924, 2849, 1615, 1501, 1450, 1420, 1244.

${ }^{1} \mathrm{H}$ NMR $\left(500 \mathrm{MHz}, \mathrm{CDCl}_{3}\right): \delta=8.20(\mathrm{~d}, J=8.5 \mathrm{~Hz}, 2 \mathrm{H}), 7.75-7.73(\mathrm{~m}$, $1 \mathrm{H}), 7.56-7.54(\mathrm{~m}, 1 \mathrm{H}), 7.33-7.31(\mathrm{~m}, 2 \mathrm{H}), 7.03(\mathrm{~d}, J=8.5 \mathrm{~Hz}, 2 \mathrm{H})$, $3.88(\mathrm{~s}, 3 \mathrm{H})$.

${ }^{13} \mathrm{C}$ NMR $\left(125 \mathrm{MHz}, \mathrm{CDCl}_{3}\right): \delta=163.3,162.6,150.8,142.1,129.7$, 124.8, 124.7, 119.7, 119.7, 114.6, 110.6, 55.6.

GC-MS (EI, $70 \mathrm{eV}): m / z=225[\mathrm{M}]^{+}$.

\section{2-(4-Fluorophenyl)benzoxazole (3ad)}

Yield: $72 \%$ (153 mg); analytical TLC on silica gel (9:1 $n$-hexane/ EtOAc); white solid; mp $102-104{ }^{\circ} \mathrm{C}$.

FT-IR (KBr): 3061, 2925, 1619, 1584, 1582, 1542, 1473, 1448, 1247 , $1225 \mathrm{~cm}^{-1}$.

${ }^{1} \mathrm{H} \mathrm{NMR}\left(500 \mathrm{MHz}, \mathrm{CDCl}_{3}\right): \delta=8.28-8.25(\mathrm{~m}, 2 \mathrm{H}), 7.78-7.76(\mathrm{~m}, 1 \mathrm{H})$, 7.59-7.57 (m, $1 \mathrm{H}), 7.37-7.35$ (m, $2 \mathrm{H}), 7.24-7.20$ (m, $2 \mathrm{H})$.

${ }^{13} \mathrm{C}$ NMR $\left(125 \mathrm{MHz}, \mathrm{CDCl}_{3}\right): \delta=165.0(\mathrm{~d}, J=251 \mathrm{~Hz}), 162.3,150.9$, $142.2,130.0(\mathrm{~d}, J=8.8 \mathrm{~Hz}), 130.0(\mathrm{~d}, J=255.1 \mathrm{~Hz}), 125.0(\mathrm{~d}, J=$ $59.5 \mathrm{~Hz}), 123.7$ (d, $J=3.3 \mathrm{~Hz}), 120.1,116.3(\mathrm{~d}, J=22 \mathrm{~Hz}), 110.7$.

GC-MS (EI, $70 \mathrm{eV}): m / z=213[\mathrm{M}]^{+}$.

\section{2-(4-tert-Butylphenyl)benzoxazole (3ae)}

Yield: $74 \%$ (186 mg); analytical TLC on silica gel (9:1 $n$-hexane/ EtOAc); white solid; mp $107-108^{\circ} \mathrm{C}$.

FT-IR (KBr): 3059, 2927, 1728, 1547, 1452, 1429, 1287, $1239 \mathrm{~cm}^{-1}$. ${ }^{1} \mathrm{H} \mathrm{NMR}\left(500 \mathrm{MHz}, \mathrm{CDCl}_{3}\right): \delta=8.29(\mathrm{dt}, J=8.5,2.0 \mathrm{~Hz}, 2 \mathrm{H}), 7.78-7.76$ (m, $1 \mathrm{H}), 7.59-7.57(\mathrm{~m}, 1 \mathrm{H}), 7.55$ (dt, $J=8.5,2.0 \mathrm{~Hz}, 2 \mathrm{H}), 7.36-7.33$ $(\mathrm{m}, 2 \mathrm{H}), 1.38(\mathrm{~s}, 9 \mathrm{H})$.

${ }^{13} \mathrm{C}$ NMR $\left(125 \mathrm{MHz}, \mathrm{CDCl}_{3}\right): \delta=163.4,155.4,150.8,142.1,127.7$, $126.1,125.1,124.7,124.4,120.0,110.7,35.2,31.3$.

GC-MS (EI, $70 \mathrm{eV}): m / z=251[\mathrm{M}]^{+}$.

\section{2-(4-Chlorophenyl)benzoxazole (3af)}

Yield: $84 \%$ (175 mg); analytical TLC on silica gel (95:5 $n$-hexane/ EtOAc); white solid; $\mathrm{mp} 148-150{ }^{\circ} \mathrm{C}$.

FT-IR (KBr): 3061, 2925, 1610, 1584, 1582, 1542, 1473, 1448, 1247 , $1225 \mathrm{~cm}^{-1}$.

${ }^{1} \mathrm{H}$ NMR $\left(500 \mathrm{MHz}, \mathrm{CDCl}_{3}\right): \delta=8.24(\mathrm{~d}, J=8.5 \mathrm{~Hz}, 2 \mathrm{H}), 8.27-8.22(\mathrm{~m}$, $2 \mathrm{H}), 7.77$ (m, $1 \mathrm{H}), 7.71-7.69$ ( $\mathrm{m}, 1 \mathrm{H}), 7.64(\mathrm{~d}, J=8.5 \mathrm{~Hz}, 2 \mathrm{H}), 7.46-$ $7.39(\mathrm{~m}, 2 \mathrm{H})$.

${ }^{13} \mathrm{C}$ NMR $\left(125 \mathrm{MHz}, \mathrm{CDCl}_{3}\right): \delta=161.73,150.81,142.08,137.23$, $129.34,128.99,125.93,125.59,124.85,120.02,110.70$.

GC-MS (EI, $70 \mathrm{eV}): m / z=229[\mathrm{M}]^{+}$.

\section{2-(2-Chlorophenyl)benzoxazole (3ah)}

Yield: $35 \%(80 \mathrm{mg})$; analytical TLC on silica gel (95:5 $n$-hexane/ EtOAc); white solid; mp $100-102{ }^{\circ} \mathrm{C}$.

FT-IR (KBr): 2925, 1608, 1584, 1569, 1533, 1470, 1452, 1343, 1237, $1185 \mathrm{~cm}^{-1}$.

${ }^{1} \mathrm{H}$ NMR $\left(500 \mathrm{MHz}, \mathrm{CDCl}_{3}\right): \delta=8.15(\mathrm{dd}, J=8.0,2.0 \mathrm{~Hz}, 1 \mathrm{H}), 7.87-7.85$ (m, $1 \mathrm{H}), 7.63-7.62(\mathrm{~m}, 1 \mathrm{H}), 7.59-7.57(\mathrm{~m}, 1 \mathrm{H}), 7.48-7.37(\mathrm{~m}, 4 \mathrm{H})$.
${ }^{13} \mathrm{C}$ NMR $\left(125 \mathrm{MHz}, \mathrm{CDCl}_{3}\right): \delta=161.1,150.8,141.8,133.7,132.1$, $132.0,131.5,127.1,126.5,125.7,124.8,120.7,110.9$.

GC-MS (EI, $70 \mathrm{eV}): m / z=229[\mathrm{M}]^{+}$.

\section{2-(2-Hydroxylphenyl)benzoxazole (3ai)}

Yield: 95\% (202 mg); analytical TLC on silica gel (9:1 $n$-hexane/ EtOAc); white solid; mp $120-122{ }^{\circ} \mathrm{C}$.

FT-IR (KBr): 2921, 2851, 1630, 1587, 1543, 1487, 1452, 1244, $1155 \mathrm{~cm}^{-1}$.

${ }^{1} \mathrm{H}$ NMR $\left(500 \mathrm{MHz}, \mathrm{CDCl}_{3}\right): \delta=11.5(\mathrm{~s}, 1 \mathrm{H}), 8.04(\mathrm{dd}, J=8.0,1.5 \mathrm{~Hz}$, $1 \mathrm{H}), 7.75-7.73(\mathrm{~m}, 1 \mathrm{H}), 7.62-7.61(\mathrm{~m}, 1 \mathrm{H}), 7.46-7.43(\mathrm{~m}, 1 \mathrm{H}), 7.41-$ $7.37(\mathrm{~m}, 2 \mathrm{H}), 7.13$ (dd, $J=8.0,1.5 \mathrm{~Hz}, 1 \mathrm{H}), 7.03-7.00(\mathrm{~m}, 1 \mathrm{H})$.

${ }^{13} \mathrm{C}$ NMR $\left(125 \mathrm{MHz}, \mathrm{CDCl}_{3}\right): \delta=133.72,127.29,125.54,125.17$, $119.72,119.43,117.59,110.82$.

GC-MS (EI, $70 \mathrm{eV}): m / z=213[\mathrm{M}]^{+}$.

\section{2-(Pyridin-4-yl)benzoxazole (3aj)}

Yield: $85 \%$ (80 mg); analytical TLC on silica gel (9:1 $n$-hexane/EtOAc); orange solid; $\mathrm{mp} 100-102{ }^{\circ} \mathrm{C}$.

FT-IR (KBr): $3460,2946,1628,878 \mathrm{~cm}^{-1}$.

${ }^{1} \mathrm{H}$ NMR $\left(500 \mathrm{MHz}, \mathrm{CDCl}_{3}\right): \delta=8.82(\mathrm{~d}, J=5.0 \mathrm{~Hz}, 2 \mathrm{H}), 8.09(\mathrm{~d}, J=$ $5.0 \mathrm{~Hz}, 2 \mathrm{H}), 7.83(\mathrm{~d}, J=7.5 \mathrm{~Hz}, 1 \mathrm{H}), 7.63(\mathrm{~d}, J=7.5 \mathrm{~Hz}, 1 \mathrm{H}), 7.42(\mathrm{~m}$, $2 \mathrm{H})$.

${ }^{13} \mathrm{C}$ NMR $\left(125 \mathrm{MHz}, \mathrm{CDCl}_{3}\right): \delta=161.0,151.1,150.9,141.9,134.6$, $126.5,125.3,121.2,120.9,111.1$.

GC-MS (EI, $70 \mathrm{eV}): m / z=196[\mathrm{M}]^{+}$.

\section{2-(2-Fluorophenyl)benzoxazole (3ag)}

Yield: 5\% (10 mg); analytical TLC on silica gel (9:1 n-hexane/EtOAc); white solid; $\mathrm{mp} 100-102{ }^{\circ} \mathrm{C}$.

FT-IR (KBr): 3061, 2925, 1719, 1584, 1582, 1542, 1473, 1448, 1247, $1225 \mathrm{~cm}^{-1}$

${ }^{1} \mathrm{H} \mathrm{NMR}\left(500 \mathrm{MHz}, \mathrm{CDCl}_{3}\right): \delta=8.24(\mathrm{td}, J=7.5,2.0 \mathrm{~Hz}, 1 \mathrm{H}), 7.85-7.83$ (m, $1 \mathrm{H}), 7.63-7.61(\mathrm{~m}, 1 \mathrm{H}), 7.55-7.51(\mathrm{~m}, 1 \mathrm{H}), 7.40-7.37(\mathrm{~m}, 2 \mathrm{H})$, 7.33-7.32 (m, $1 \mathrm{H}), 7.30-7.27(\mathrm{~m}, 1 \mathrm{H})$.

${ }^{13} \mathrm{C}$ NMR $\left(125 \mathrm{MHz}, \mathrm{CDCl}_{3}\right): \delta=162.0,156.0,150.7,141.9,133.25$ (d, $J=8.6 \mathrm{~Hz}), 130.7(\mathrm{~d}, J=1.1 \mathrm{~Hz}), 125.6,124.8,124.63(\mathrm{~d}, J=3.8 \mathrm{~Hz})$, $120.52,117.32(\mathrm{~d}, J=21.3 \mathrm{~Hz}), 115.7(\mathrm{~d}, J=10.4 \mathrm{~Hz}), 110.8$.

GC-MS (EI, $70 \mathrm{eV}): m / z=213[\mathrm{M}]^{+}$.

\section{5-Methyl-2-phenylbenzoxazole (3ba)}

Yield: $71 \%(148 \mathrm{mg})$; analytical TLC on silica gel (9:1 $n$-hexane/ EtOAc); white solid; mp $112-115^{\circ} \mathrm{C}$.

FT-IR (KBr): 2918, 1626, 1552, 1445, $1263 \mathrm{~cm}^{-1}$.

${ }^{1} \mathrm{H}$ NMR $\left(500 \mathrm{MHz}, \mathrm{CDCl}_{3}\right): \delta=8.26-8.24(\mathrm{~m}, 2 \mathrm{H}), 7.56(\mathrm{~s}, 1 \mathrm{H}), 7.53-$ $7.51(\mathrm{~m}, 3 \mathrm{H}), 7.45$ (d, $J=8.5 \mathrm{~Hz}, 1 \mathrm{H}), 7.16(\mathrm{~d}, J=8.5 \mathrm{~Hz}, 1 \mathrm{H}), 2.49$ (s, $3 \mathrm{H})$.

${ }^{13} \mathrm{C}$ NMR $\left(125 \mathrm{MHz}, \mathrm{CDCl}_{3}\right): \delta=163.3,149.2,142.5,134.5,131.5$, $129.0,127.7,127.5,126.4,120.1,110.1,21.6$

GC-MS (EI, $70 \mathrm{eV}): m / z=209[\mathrm{M}]^{+}$.

\section{5-Methyl-2-(4-methyl-phenyl)benzoxazole (3bb)}

Yield: $73 \%(163 \mathrm{mg})$; analytical TLC on silica gel (9:1 $n$-hexane/ EtOAc); white solid; mp $138-140{ }^{\circ} \mathrm{C}$.

FT-IR (KBr): 3304, 2921, 2856, 1616, 1555, 1499, 1332, $1262 \mathrm{~cm}^{-1}$. 
${ }^{1} \mathrm{H}$ NMR $\left(500 \mathrm{MHz}, \mathrm{CDCl}_{3}\right): \delta=8.13(\mathrm{~d}, J=8.0 \mathrm{~Hz}, 2 \mathrm{H}), 7.54(\mathrm{~s}, 1 \mathrm{H})$, $7.43(\mathrm{~d}, J=8.0 \mathrm{~Hz}, 1 \mathrm{H}), 7.33(\mathrm{~d}, J=8.0 \mathrm{~Hz}, 2 \mathrm{H}), 7.13(\mathrm{~d}, J=8.0 \mathrm{~Hz}$, $1 \mathrm{H}), 2.48$ (s, $3 \mathrm{H}), 2.44$ (s, $3 \mathrm{H})$.

${ }^{13} \mathrm{C}$ NMR $\left(125 \mathrm{MHz}\right.$, acetone- $\left.d_{6}\right): \delta=163.2,149.2,142.7,142.3,134.5$, $129.9,127.5,126.3,124.8,119.9,110.1,20.9,20.8$.

GC-MS (EI, $70 \mathrm{eV}): m / z=223[\mathrm{M}]^{+}$.

\section{5-Methyl-2-(4-tert-butylphenyl)benzoxazole (3be)}

Yield: $67 \%(178 \mathrm{mg})$; analytical TLC on silica gel (9:1 $n$-hexane/ EtOAc); white solid; mp $148-150{ }^{\circ} \mathrm{C}$.

FT-IR (KBr): 3107, 2963, 2930, 2871, 1729, 1621, 1532, 1460, $1269 \mathrm{~cm}^{-1}$.

${ }^{1} \mathrm{H}$ NMR $\left(500 \mathrm{MHz}, \mathrm{CDCl}_{3}\right): \delta=8.17(\mathrm{~d}, J=8.5 \mathrm{~Hz}, 2 \mathrm{H}), 7.55-7.53(\mathrm{~m}$, $2 \mathrm{H}), 7.44$ (d, $J=8.5 \mathrm{~Hz}, 1 \mathrm{H}), 7.14(\mathrm{dd}, J=8.5,1.0 \mathrm{~Hz}, 1 \mathrm{H}), 2.48(\mathrm{~s}$, $3 \mathrm{H}), 1.38(\mathrm{~s}, 9 \mathrm{H})$.

${ }^{13} \mathrm{C}$ NMR $\left(125 \mathrm{MHz}\right.$, acetone- $\left.d_{6}\right): \delta=162.9,155.0,149.0,142.5,134.3$, 127.2, 126.1, 126.0, 124.6, 119.7, 109.9, 34.7, 30.5, 20.5.

GC-MS (EI, $70 \mathrm{eV}): m / z=265[\mathrm{M}]^{+}$.

\section{5-Methyl-2-(4-chlorophenyl)benzoxazole (3bf)}

Yield: $20 \%$ (48 mg); analytical TLC on silica gel (9:1 $n$-hexane/EtOAc); white solid; $\mathrm{mp} 150-151{ }^{\circ} \mathrm{C}$.

FT-IR (KBr): 3050, 2957, 2849, 1615, 1501, 1450, 1420, $1287 \mathrm{~cm}^{-1}$.

${ }^{1} \mathrm{H} \mathrm{NMR}\left(500 \mathrm{MHz}, \mathrm{CDCl}_{3}\right): \delta=8.15(\mathrm{~d}, J=8.5 \mathrm{~Hz}, 2 \mathrm{H}), 7.53(\mathrm{~s}, 1 \mathrm{H})$, $7.47(\mathrm{~d}, J=8.5 \mathrm{~Hz}, 2 \mathrm{H}), 7.42(\mathrm{~d}, J=8.5 \mathrm{~Hz}, 1 \mathrm{H}), 7.16(\mathrm{~d}, J=8.5 \mathrm{~Hz}$, $1 \mathrm{H}), 2.48(\mathrm{~s}, 3 \mathrm{H})$.

${ }^{13} \mathrm{C}$ NMR $\left(125 \mathrm{MHz}, \mathrm{CDCl}_{3}\right): \delta=162.3,149.2,142.4,137.7,134.7$, $129.3,128.9,126.6,126.0,120.1,110.1,21.6$.

GC-MS (EI, $70 \mathrm{eV}): m / z=243[\mathrm{M}]^{+}$.

\section{5-Methyl-2-(pyridin-4-yl)benzoxazole (3bi)}

Yield: 45\% (94 mg); analytical TLC on silica gel (9:1 $n$-hexane/EtOAc); orange solid; mp $129-130^{\circ} \mathrm{C}$.

FT-IR (KBr): 3406, 2921, 1612, 1537, 1414, 1344, 1068, $808 \mathrm{~cm}^{-1}$.

${ }^{1} \mathrm{H}$ NMR $\left(500 \mathrm{MHz}, \mathrm{CDCl}_{3}\right): \delta=8.80(\mathrm{~d}, J=5.5 \mathrm{~Hz}, 2 \mathrm{H}), 8.07$ (dd, $J=5.5$, $1.5 \mathrm{~Hz}, 2 \mathrm{H}), 7.60(\mathrm{~s}, 1 \mathrm{H}), 7.49(\mathrm{~d}, J=8.5 \mathrm{~Hz}, 1 \mathrm{H}), 7.24(\mathrm{dd}, J=8.5$, $1.0 \mathrm{~Hz}, 1 \mathrm{H}), 2.51(\mathrm{~s}, 3 \mathrm{H})$

${ }^{13} \mathrm{C}$ NMR $\left(125 \mathrm{MHz}, \mathrm{CDCl}_{3}\right): \delta=160.8,150.7,149.3,142.1,135.2$, 134.8, 127.7, 121.1, 120.6, 110.4, 21.6.

GC-MS (EI, $70 \mathrm{eV}): m / z=210[\mathrm{M}]^{+}$.

\section{5-Chloro-2-phenylbenzoxazole (3ca)}

Yield: 92\% (210 mg); analytical TLC on silica gel (9:1 $n$-hexane/ EtOAc); white solid; mp 102-104 ${ }^{\circ} \mathrm{C}$.

FT-IR (KBr): 3061, 1612, 1551, 1443, 1333, $1265 \mathrm{~cm}^{-1}$.

${ }^{1} \mathrm{H}$ NMR $\left(500 \mathrm{MHz}, \mathrm{CDCl}_{3}\right): \delta=8.24-8.22(\mathrm{~m}, 2 \mathrm{H}), 7.75(\mathrm{~d}, J=1.5 \mathrm{~Hz}$, $1 \mathrm{H}), 7.55-7.53(\mathrm{~m}, 3 \mathrm{H}), 7.50-7.49(\mathrm{~m}, 1 \mathrm{H}), 7.32$ (dd, $J=8.5,1.5 \mathrm{~Hz}$, $1 \mathrm{H})$.

${ }^{13} \mathrm{C}$ NMR $\left(125 \mathrm{MHz}\right.$, acetone- $\left.d_{6}\right): \delta=165.3,150.6,144.5,133.1,130.7$, $130.2,128.6,127.7,126.4,120.7,112.9$.

GC-MS (EI, $70 \mathrm{eV}): m / z=229[\mathrm{M}]^{+}$.

\section{5-Chloro-2-(4-methylphenyl)benzoxazole (3cb)}

Yield: $85 \%$ (206 mg); analytical TLC on silica gel (9:1 $n$-hexane/ EtOAc); white solid; mp $148-150{ }^{\circ} \mathrm{C}$.
FT-IR (KBr): 2953, 1610, 1551, 1479, 1448, $1258 \mathrm{~cm}^{-1}$.

${ }^{1} \mathrm{H}$ NMR $\left(500 \mathrm{MHz}, \mathrm{CDCl}_{3}\right): \delta=8.12(\mathrm{~d}, J=8.5 \mathrm{~Hz}, 2 \mathrm{H}), 7.72(\mathrm{~d}, J=$ $2.0 \mathrm{~Hz}, 1 \mathrm{H}$ ), 7.47 (d, $J=8.5 \mathrm{~Hz}, 1 \mathrm{H}$ ), 7.33 (d, $J=8.5 \mathrm{~Hz}, 2 \mathrm{H}$ ), 7.30 (dd, $J=8.5,2.0 \mathrm{~Hz}, 1 \mathrm{H}), 2.44(\mathrm{~s}, 3 \mathrm{H})$

${ }^{13} \mathrm{C}$ NMR $\left(125 \mathrm{MHz}\right.$, acetone- $\left.d_{6}\right): \delta=165.6,150.5,144.6,143.8,130.8$, 130.6, 128.6, 126.2, 125.0, 120.5, 112.8, 21.7.

GC-MS (EI, $70 \mathrm{eV}): m / z=243[\mathrm{M}]^{+}$.

\section{5-Chloro-2-(4-fluorophenyl)benzoxazole (3cd)}

Yield: $80 \%$ (197 mg); analytical TLC on silica gel (9:1 $n$-hexane/ EtOAc); white solid; mp $156-157^{\circ} \mathrm{C}$.

FT-IR (KBr): 3061, 2922, 1610, 1551, 1481, 1450, 1331, 1260, 1227.

${ }^{1} \mathrm{H}$ NMR $\left(500 \mathrm{MHz}, \mathrm{CDCl}_{3}\right): \delta=8.25-8.23(\mathrm{~m}, 2 \mathrm{H}), 7.73(\mathrm{~d}, J=2.0 \mathrm{~Hz}$, $1 \mathrm{H}), 7.49(\mathrm{~d}, J=8.5 \mathrm{~Hz}, 1 \mathrm{H}), 7.32(\mathrm{dd}, J=8.5,2.0 \mathrm{~Hz}, 1 \mathrm{H}), 7.24-7.20$ (m, $2 \mathrm{H})$.

${ }^{13} \mathrm{C}$ NMR $\left(125 \mathrm{MHz}\right.$, acetone- $\left.d_{6}\right): \delta=166.0(\mathrm{~d}, J=250 \mathrm{~Hz}), 164.3$, $150.5,144.3,131.1$ (d, $J=9.0 \mathrm{~Hz}), 130.6,126.3,124.2,120.5,117.2$ (d, $J=22.4 \mathrm{~Hz}), 112.7$.

GC-MS (EI, $70 \mathrm{eV}): m / z=247[\mathrm{M}]^{+}$

\section{5-Chloro-2-(4-tert-butylphenyl)benzoxazole (3ce)}

Yield: $90 \%$ (256 mg); analytical TLC on silica gel (9:1 $n$-hexane/ EtOAc); white solid; $\mathrm{mp} 137-138^{\circ} \mathrm{C}$.

FT-IR (KBr): 2957, 2902, 2866, 1611, 1552, 1493, 1457, $1262 \mathrm{~cm}^{-1}$.

${ }^{1} \mathrm{H}$ NMR $\left(500 \mathrm{MHz}, \mathrm{CDCl}_{3}\right): \delta=8.16(\mathrm{~d}, J=9.0 \mathrm{~Hz}, 2 \mathrm{H}), 7.73(\mathrm{~d}, J=$ $2.0 \mathrm{~Hz}, 1 \mathrm{H}), 7.54$ (d, $J=9.0 \mathrm{~Hz}, 2 \mathrm{H}), 7.48(\mathrm{~d}, J=9.0 \mathrm{~Hz}, 1 \mathrm{H}), 7.30$ (dd, $J=9.0,2.0 \mathrm{~Hz}, 1 \mathrm{H}), 1.38(\mathrm{~s}, 9 \mathrm{H})$.

${ }^{13} \mathrm{C}$ NMR $\left(125 \mathrm{MHz}\right.$, acetone- $\left.d_{6}\right): \delta=165.5,156.7,150.6,144.6,130.6$, 128.6, 127.2, 126.2, 125.0, 120.5, 112.8, 35.9, 31.5.

GC-MS (EI, $70 \mathrm{eV}): m / z=285[\mathrm{M}]^{+}$.

\section{5-Chloro-2-(4-chlorophenyl)benzoxazole (3cf)}

Yield: 55\% (144 mg); analytical TLC on silica gel (9:1 $n$-hexane/ EtOAc); white solid; mp $195-197^{\circ} \mathrm{C}$.

FT-IR (KBr): 2913, 1727, 1550, 1448, $1245 \mathrm{~cm}^{-1}$.

${ }^{1} \mathrm{H}$ NMR $\left(500 \mathrm{MHz}, \mathrm{CDCl}_{3}\right): \delta=8.16(\mathrm{~d}, J=8.5 \mathrm{~Hz}, 2 \mathrm{H}), 7.74(\mathrm{~d}, J=$ $1.5 \mathrm{~Hz}, 1 \mathrm{H}$ ), 7.51-7.48 (m, $3 \mathrm{H}$ ), 7.33 (dd, $J=8.5,1.5 \mathrm{~Hz}, 1 \mathrm{H}$ ).

${ }^{13} \mathrm{C}$ NMR $\left(125 \mathrm{MHz}\right.$, acetone- $\left.d_{6}\right): \delta=149.6,143.4,137.7,129.8,129.4$, 129.2, 125.7, 125.5, 119.7, 111.9.

GC-MS (EI, $70 \mathrm{eV}): m / z=263[\mathrm{M}]^{+}$.

\section{5-Chloro-2-(pyridin-4-yl)benzoxazole (3ci)}

Yield: $83 \%$ (190 mg); analytical TLC on silica gel (9:1 $n$-hexane/ EtOAc); orange solid; $\mathrm{mp} 152-153{ }^{\circ} \mathrm{C}$.

FT-IR (KBr): 3431, 2922, 1612, 1568, 1539, 1449, 1057, $874 \mathrm{~cm}^{-1}$

${ }^{1} \mathrm{H}$ NMR $\left(500 \mathrm{MHz}, \mathrm{CDCl}_{3}\right): \delta=8.83(\mathrm{~d}, J=5.5 \mathrm{~Hz}, 2 \mathrm{H}), 8.07(\mathrm{dd}, J=4.5$, $1.5 \mathrm{~Hz}, 2 \mathrm{H}), 7.81(\mathrm{~d}, J=2.0 \mathrm{~Hz}, 1 \mathrm{H}), 7.56(\mathrm{~d}, J=8.5 \mathrm{~Hz}, 1 \mathrm{H}), 7.41(\mathrm{dd}$, $J=8.5,2.0 \mathrm{~Hz}, 1 \mathrm{H}$ ).

${ }^{13} \mathrm{C}$ NMR $\left(125 \mathrm{MHz}, \mathrm{CDCl}_{3}\right): \delta=162.0,150.8,149.6,143.0,134.2$, 130.9, 126.9, 121.3, 120.8, 111.9.

GC-MS (EI, $70 \mathrm{eV}): m / z=230[\mathrm{M}]^{+}$

\section{2-Phenylbenzimidazole (3da)}

Yield: 70\% (136 mg); analytical TLC on silica gel (9:1 $n$-hexane/ EtOAc); white solid; mp 293-294 ${ }^{\circ} \mathrm{C}$. 
FT-IR (KBr): 3411, 1540, 1450, 1408, 967, $774 \mathrm{~cm}^{-1}$.

${ }^{1} \mathrm{H}$ NMR $\left(500 \mathrm{MHz}\right.$, DMSO- $\left.d_{6}\right): \delta=8.18(\mathrm{~d}, J=7.5 \mathrm{~Hz}, \mathrm{H}), 7.60-7.54(\mathrm{~m}$, $4 \mathrm{H})$, 7.51-7.48 (m, $1 \mathrm{H}), 7.22-7.19(\mathrm{~m}, 2 \mathrm{H})$.

${ }^{13} \mathrm{C}$ NMR $\left(125 \mathrm{MHz}\right.$, DMSO- $\left.d_{6}\right): \delta=151.2,130.2,129.8,128.9,126.4$, 122.1 .

GC-MS (EI, $70 \mathrm{eV}): m / z=194[\mathrm{M}]^{+}$.

\section{2-(4-Methoxyphenyl)benzimidazole (3dc)}

Yield: 65\% (145 mg); analytical TLC on silica gel (9:1 $n$-hexane/ EtOAc); white solid; $\mathrm{mp} 293-294{ }^{\circ} \mathrm{C}$.

FT-IR (KBr): 3395, 3055, 1452, 1406, 1274, $741 \mathrm{~cm}^{-1}$.

${ }^{1} \mathrm{H}$ NMR (500 MHz, DMSO- $\left.d_{6}\right): \delta=8.49(\mathrm{~s}, 1 \mathrm{H}), 8.05(\mathrm{~d}, J=7.5 \mathrm{~Hz}$, $1 \mathrm{H}), 7.88(\mathrm{~d}, J=9 \mathrm{~Hz}, 2 \mathrm{H}), 7.81(\mathrm{~d}, J=8.0 \mathrm{~Hz}, 1 \mathrm{H}), 7.47-7.41(\mathrm{~m}, 2 \mathrm{H})$, $7.17(\mathrm{~d}, J=8.5 \mathrm{~Hz}, 2 \mathrm{H}), 3.90(\mathrm{~s}, 3 \mathrm{H})$.

${ }^{13} \mathrm{C}$ NMR $\left(125 \mathrm{MHz}, \mathrm{DMSO}-d_{6}\right): \delta=166.4,163.2,144.3,143.7,132.4$, 132.1, 131.6, 125.1, 124.7, 124.5, 120.1, 115.0, 114.4, 55.7.

GC-MS (EI, $70 \mathrm{eV}): m / z=224[\mathrm{M}]^{+}$.

\section{6-Methoxy-2phenylbenzimidazole (3ea)}

Yield: $85 \%$ (190 mg); analytical TLC on silica gel (9:1 $n$-hexane/ EtOAc); white solid; $\mathrm{mp}>300{ }^{\circ} \mathrm{C}$.

${ }^{1} \mathrm{H} \mathrm{NMR}\left(500 \mathrm{MHz}, \mathrm{CDCl}_{3}\right): \delta=8.03(\mathrm{dd}, J=8.0,1.5 \mathrm{~Hz}, 2 \mathrm{H}), 7.51(\mathrm{~d}, J=$ $9.0 \mathrm{~Hz}, 1 \mathrm{H}), 7.47-7.42(\mathrm{~m}, 3 \mathrm{H}), 7.08(\mathrm{~d}, J=1.5 \mathrm{~Hz}, 1 \mathrm{H}), 6.90$ (dd, $J=$ 9.0, $1.5 \mathrm{~Hz}, 1 \mathrm{H}), 3.83(\mathrm{~s}, 3 \mathrm{H})$.

${ }^{13} \mathrm{C}$ NMR $\left(125 \mathrm{MHz}, \mathrm{CDCl}_{3}\right): \delta=171.2,156.8,151.2,130.0,129.8$, 129.1, 126.3, 55.8.

GC-MS (EI, $70 \mathrm{eV}): m / z=224[\mathrm{M}]^{+}$.

\section{6-Methoxy-2-(4-methoxyphenyl)benzimidazole (3ec)}

Yield: 68\% (173 mg); analytical TLC on silica gel (9:1 $n$-hexane/ EtOAc); orange solid; $\mathrm{mp}>300^{\circ} \mathrm{C}$.

${ }^{1} \mathrm{H} \mathrm{NMR}\left(500 \mathrm{MHz}, \mathrm{CDCl}_{3}\right): \delta=7.93(\mathrm{~s}, 2 \mathrm{H}), 7.36(\mathrm{~s}, 1 \mathrm{H}), 6.95(\mathrm{~s}, 1 \mathrm{H})$, $6.84(\mathrm{~s}, 2 \mathrm{H}), 6.74(\mathrm{~s}, 1 \mathrm{H}), 3.72(\mathrm{~s}, 6 \mathrm{H})$.

${ }^{13} \mathrm{C}$ NMR $\left(125 \mathrm{MHz}, \mathrm{CDCl}_{3}\right): \delta=161.0,156.5,151.6,128.0,122.3$, 115.6, 114.4, 113.9, 112.0, 97.6, 55.7, 55.3.

GC-MS (EI, $70 \mathrm{eV}): m / z=254[\mathrm{M}]^{+}$.

\section{H-Benzo[c][1,2]dithiole-3-thione}

Obtained as a by-product in the reaction of 2-nitrophenol and 2-fluorobenzaldehyde.

Analytical TLC on silica gel (9:1 $n$-hexane/EtOAc); orange solid; mp 96-98 ${ }^{\circ} \mathrm{C}$.

${ }^{1} \mathrm{H}$ NMR $\left(500 \mathrm{MHz}, \mathrm{CDCl}_{3}\right): \delta=8.22(\mathrm{~d}, J=8.2 \mathrm{~Hz}, 1 \mathrm{H}), 7.73(\mathrm{~d}, J=$ $8.2 \mathrm{~Hz}, 1 \mathrm{H}), 7.70-7.66(\mathrm{~m}, 1 \mathrm{H}), 7.47-7.44(\mathrm{~m}, 1 \mathrm{H})$.

${ }^{13} \mathrm{C}$ NMR $\left(125 \mathrm{MHz}, \mathrm{CDCl}_{3}\right): \delta=216.9,152.8,140.8,132.7,128.6$, $126.0,124.3$.

GC-MS (EI, $70 \mathrm{eV}): m / z=184[\mathrm{M}]^{+}$.

\section{Acknowledgment}

We thank Duy-Khiem Nguyen Chau (North Carolina State University, USA) and MSc Mai Hoang Ngoc Do (IPH-HCM) for their help.

\section{Supporting Information}

Supporting information for this article is available online at https://doi.org/10.1055/s-0039-1690823.

\section{References}

(1) (a) Marion, P.; Bernela, B.; Piccirilli, A.; Estrine, B.; Patouillard, N.; Guilbot, J.; Jérôme, F. Green Chem. 2017, 19, 4973. (b) Curzons, A. D.; Constable, D. J. C.; Mortimer, D. N.; Cunningham, V. L. Green Chem. 2001, 3, 1. (c) Sheldon, R. A. Green Chem. 2005, 7, 267. (d) Gui, Q.-W.; He, X.; Wang, W.; Zhou, H.; Dong, Y.; Wang, N.; Tang, J.-X.; Cao, Z.; He, W.-M. Green Chem. 2020, 22, 118. (e) Cao, Z.; Zhu, Q.; Lin, Y.-W.; He, W.-M. Chin. Chem. Lett. 2019, 30, 2132.

(2) (a) Tran, P. H.; Bui, T.-P. T.; Lam, X.-Q. B.; Nguyen, X.-T. T. RSC Adv. 2018, 8, 36392. (b) Tran, P. H.; Nguyen, X.-T. T.; Chau, D.-K. N. Asian J. Org. Chem. 2018, 7, 232. (c) Nguyen, H. T.; Le, N.-P. T.; Chau, D.-K. N.; Tran, P. H. RSC Adv. 2018, 8, 35681. (d) Lu, L.-H.; Wang, Z.; Xia, W.; Cheng, P.; Zhang, B.; Cao, Z.; He, W.-M. Chin. Chem. Lett. 2019, 30, 1237. (e) Xie, L.-Y.; Jiang, L.-L.; Tan, J.-X.; Wang, Y.; Xu, X.-Q.; Zhang, B.; Cao, Z.; He, W.-M. ACS Sustainable Chem. Eng. 2019, 7, 14153.

(3) Nguyen, T. B. Adv. Synth. Catal. 2017, 359, 1066.

(4) (a) Nguyen, T. B. Asian J. Org. Chem. 2017, 6, 477. (b) Nguyen, T. B.; Retailleau, P. Org. Lett. 2018, 20, 186. (c) Nguyen, T. B.; Retailleau, P. Green Chem. 2018, 20, 387.

(5) (a) Nguyen, T. B.; Pasturaud, K.; Ermolenko, L.; Al-Mourabit, A. Org. Lett. 2015, 17, 2562. (b) Li, B.; Ni, P.; Huang, H.; Xiao, F.; Deng, G.-J. Adv. Synth. Catal. 2017, 359, 4300. (c) Nguyen, L. A.; Ngo, Q. A.; Retailleau, P.; Nguyen, T. B. Green Chem. 2017, 19, 4289. (d) Nguyen, T. B.; Retailleau, P. Org. Lett. 2017, 19, 3879. (e) Nguyen, T. B.; Retailleau, P. Org. Lett. 2017, 19, 3887. (f) Nguyen, T. B.; Retailleau, P. Org. Lett. 2017, 19, 4858. (g) Nguyen, T. B.; Retailleau, P. Adv. Synth. Catal. 2017, 359, 3843. (h) Ni, P.; Li, B.; Huang, H.; Xiao, F.; Deng, G.-J. Green Chem. 2017, 19, 5553.

(6) (a) Demmer, C. S.; Bunch, L. Eur. J. Med. Chem. 2015, 97, 778. (b) Oh, Y. J.; Kim, D.; Oh, S.; Jang, E. J.; Won, H. Y.; Jeong, H.; Jeong, M. G.; Choo, H. P.; Hwang, E. S. Sci. Rep. 2017, 7, 42144. (c) Nam, M. H.; Park, M.; Park, H.; Kim, Y.; Yoon, S.; Sawant, V. S.; Choi, J. W.; Park, J. H.; Park, K. D.; Min, S. J.; Lee, C. J.; Choo, H. ACS Chem. Neurosci. 2017, 8, 1519. (d) Seth, K.; Garg, S. K.; Kumar, R.; Purohit, P.; Meena, V. S.; Goyal, R.; Banerjee, U. C.; Chakraborti, A. K. ACS Med. Chem. Lett. 2014, 5, 512. (e) Kuroda, K.; Tsuyumine, S.; Kodama, T. Org. Process Res. Dev. 2016, 20, 1053. (f) Zhang, W.; Liu, J.; Macho, J. M.; Jiang, X.; Xie, D.; Jiang, F.; Liu, W.; Fu, L. Eur. J. Med. Chem. 2017, 126, 7. (g) Kim, D.; Won, H. Y.; Hwang, E. S.; Kim, Y. K.; Choo, H. P. Bioorg. Med. Chem. 2017, 25, 3127. (h) Chanda, K.; Rajasekhar, S.; Maiti, B. Synlett 2017, 28, 521. (i) Gorla, S. K.; Kavitha, M.; Zhang, M.; Chin, J. E.; Liu, X.; Striepen, B.; Makowska-Grzyska, M.; Kim, Y.; Joachimiak, A.; Hedstrom, L.; Cuny, G. D. J. Med. Chem. 2013, 56, 4028.

(7) (a) Ziarati, A.; Sobhani-Nasab, A.; Rahimi-Nasrabadi, M.; Ganjali, M. R.; Badiei, A. J. Rare Earths 2017, 35, 374. (b) Szlachcic, P.; Fedorchuk, A. A.; Danel, A.; Jarosz, B.; El Naggar, A. M.; Albassam, A. A.; Wojciechowski, A.; Gondek, E.; Uchacz, T.; Stadnicka, K.; Lakshminarayana, G.; Kityk, I. V. Dyes Pigm. 2017, 141, 333. (c) Azizian, J.; Torabi, P.; Noei, J. Tetrahedron Lett. 2016, 57, 185. (d) Maleki, B.; Baghayeri, M.; Vahdat, S. M.; Mohammadzadeh, A.; Akhoondi, S. RSC Adv. 2015, 5, 46545. 
Synthesis

M.-H. D. Dang et al.

(e) Kawashita, Y.; Nakamichi, N.; Kawabata, H.; Hayashi, M. Org. Lett. 2003, 5, 3713. (f) Cho, Y. H.; Lee, C.-Y.; Ha, D.-C.; Cheon, C.H. Adv. Synth. Catal. 2012, 354, 2992.

(8) Nguyen, L. H. T.; Nguyen, T. T.; Nguyen, H. L.; Doan, T. L. H.; Tran, P. H. Catal. Sci. Technol. 2017, 7, 4346.
(9) Nguyen, Q. T.; Hang, A.-H. T.; Nguyen, T.-L. H.; Chau, D.-K. N.; Tran, P. H. RSC Adv. 2018, 8, 11834.

(10) Yu, C.; Guo, X.; Xi, Z.; Muzzio, M.; Yin, Z.; Shen, B.; Li, J.; Seto, C. T.; Sun, S. J. Am. Chem. Soc. 2017, 139, 5712.

(11) Nguyen, T. B.; Ermolenko, L.; Retailleau, P.; Al-Mourabit, A. Angew. Chem. Int. Ed. 2014, 53, 13808. 